\title{
Distinguishing between symbiotic stars and planetary nebulae
}

\author{
K. Iłkiewicz and J. Mikołajewska
}

\begin{abstract}
Nicolaus Copernicus Astronomical Center, Polish Academy of Sciences, ul. Bartycka 18, 00-716 Warsaw, Poland e-mail: ilkiewicz@camk.edu.pl
\end{abstract}

Received 3 July 2017 / Accepted 22 August 2017

\begin{abstract}
Context. The number of known symbiotic stars (SySt) is still significantly lower than their predicted population. One of the main problems in finding the total population of SySt is the fact that their spectrum can be confused with other objects, such as planetary nebulae (PNe) or dense H II regions. This problem is reinforced by the fact that in a significant fraction of established SySt the emission lines used to distinguish them from other objects are not present.

Aims. We aim at finding new diagnostic diagrams that could help separate SySt from PNe. Additionally, we examine a known sample of extragalactic PNe for candidate SySt.

Methods. We employed emission line fluxes of known SySt and PNe from the literature.

Results. We found that among the forbidden lines in the optical region of spectrum, only the [O III] and [N II] lines can be used as a tool for distinguishing between SySt and PNe, which is consistent with the fact that they have the highest critical densities. The most useful diagnostic that we propose is based on He I lines, which are more common and stronger in SySt than forbidden lines. All these useful diagnostic diagrams are electron density indicators that better distinguish PNe and ionized symbiotic nebulae. Moreover, we found six new candidate SySt in the Large Magellanic Cloud and one in M 81. If confirmed, the candidate in M 81 would be the farthest known SySt thus far.
\end{abstract}

Key words. planetary nebulae: general - binaries: general - galaxies: individual: LMC, SMC, M 33, M 81, NGC 300 binaries: symbiotic - binaries: symbiotic

\section{Introduction}

Symbiotic stars (SySt) are interacting binaries with the longest orbital periods. In these systems an evolved, cool star is transferring mass to a much hotter and more luminous compact companion. The mass accretor is typically a white dwarf (WD), but in some cases a neutron star (NS) is observed. The mass donor is a normal red giant (RG) in S-type (stellar) SySt, or a Mira surrounded by a warm dust shell in D-type (dust) systems. In $\mathrm{D}^{\prime}$ type (dusty) SySt there is an F- or G-type cool giant surrounded by a dust shell. SySt are good laboratories for binary interaction and evolution because they show such phenomena as jets, accretion/excretion discs, nova outbursts and interacting winds. Moreover, they are promising candidates for progenitors of type Ia supernovae (see e.g., Hachisu et al. 1999; Lü et al. 2009; Di Stefano 2010; Chen et al. 2011). The most recent review of properties of these systems is presented by Mikołajewska (2012).

Thus far $~ 300$ SySt have been discovered in the Milky Way (MW Belczyński et al. 2000; Miszalski et al. 2013; Miszalski \& Mikołajewska 2014; Rodríguez-Flores et al. 2014; Li et al. 2015; Baella et al. 2016, and references therein). This number is considerably lower than the predicted number of SySt in the Galaxy, ranging from 3000 (Allen 1984) up to $4 \times 10^{5}$ (Magrini et al. 2003). This, combined with the fact that the distance to most of the Galactic SySt is not known, hinders the study of their properties. The situation is improving thanks to a growing number of known SySt in the Local Group of Galaxies (LGGS, Belczyński et al. 2000; Gonçalves et al. 2008; Kniazev et al. 2009; Miszalski et al. 2014; Mikołajewska et al. 2014, 2017; Gonçalves et al. 2015), which in future may lead to observing a full sample of SySt in one of the galaxies.
The current definition of a SySt used in the literature includes the presence of a late-type giant feature in the spectrum, strong emission lines of $\mathrm{HI}$ and He I as well as the presence of emission lines with a high ionization potential (Kenyon 1986). Mikołajewska et al. (2017) have recently highlighted that such a method of classification of SySt in LGGS can be hindered by contamination of the spectrum by diffuse interstellar gas (DIG). Moreover, in D-type systems, where the molecular bands of the RG cannot be detected, the SySt can be mistaken for a planetary nebula (PN). In fact, a large fraction of the SySt have originally been classified as PNe (see e.g., Belczyński et al. 2000, and references therein). Thus far the most reliable criterion for SySt was the presence of O VI Raman scattered 6825 and $7082 \AA$ lines. However, this line is present only in $\sim 50 \%$ of SySt (Allen 1980).

Gutierrez-Moreno et al. (1995) proposed a diagnostic diagram using [O III] and Balmer emission lines, that have been successfully employed to classify new SySt. The [O III] lines are not always detected in SySt, so the Gutierrez-Moreno et al. (1995) criterion is not always applicable. Moreover, some SySt have been observed in the region dominated by young PNe (Baella 2010). In some studies criteria based on infrared colors have been suggested (Schmeja \& Kimeswenger 2001; RodríguezFlores et al. 2014), however these criteria are only applicable in the Galaxy. Because of the above restrictions, in this paper, we explore the possibility of using other emission lines for classifying new SySt. Similar comparisons of the emission line ratios in SySt and PNe have been carried out in the past (e.g., GutierrezMoreno et al. 1986), however to our knowledge this is the first study focused on new criteria and using such a big sample of data. Moreover, in this study we examine a known sample of extragalactic PNe in order to detect misclassified SySt among them. 

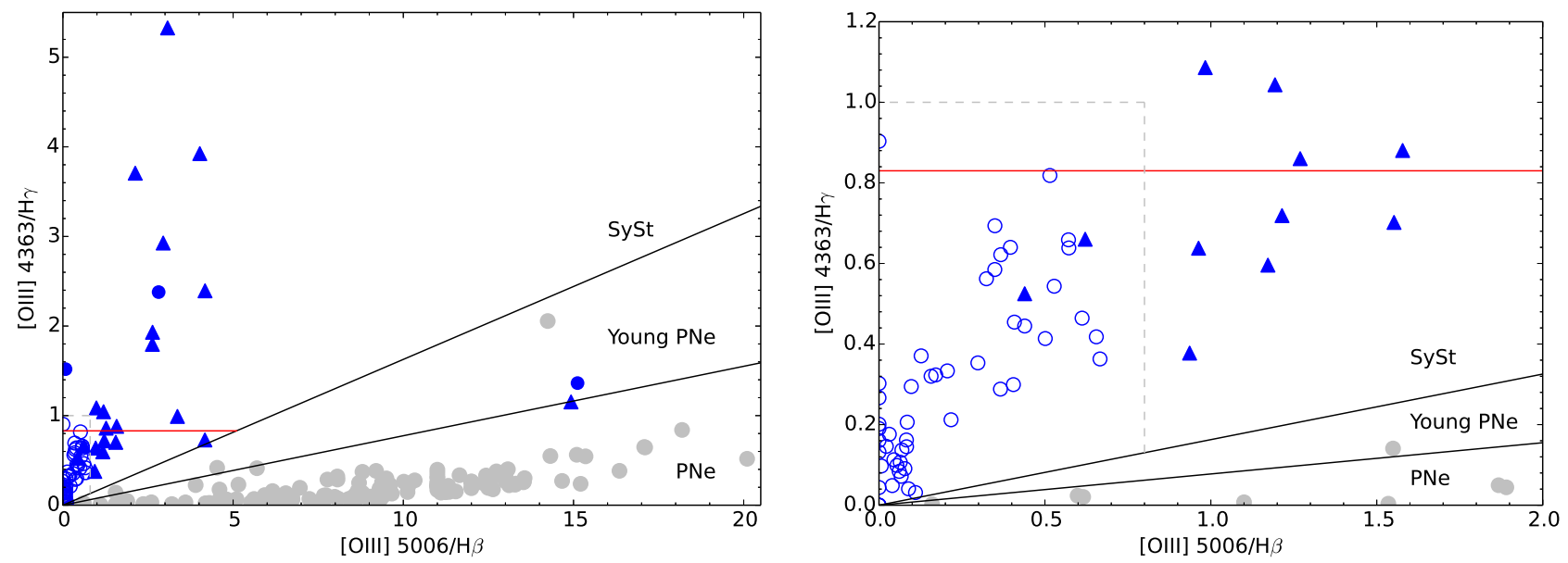

Fig. 1. [O III] diagnostic diagram of Gutierrez-Moreno et al. (1995) for SySt (blue symbols) and PNe (gray symbols) in the MW. The S-type SySt are marked with open circles, the D'-type SySt with full circles, and D-type SySt with triangles. The red line marks Kniazev et al. (2008) criterion for distinguishing between S- and D-type SySt. The gray lines indicate the regions of S- and D-type SySt proposed in this work. Left and right panel: same diagram, but with different zoom.

\section{Observations}

In order to examine the possibility of new diagnostics for SySt we used emission line fluxes of PNe and SySt from the Milky Way (MW), where the objects are well studied and contamination of the spectra by DIG is usually not a concern. In the case of PNe, the emission line fluxes were taken from Górny et al. (2004), Górny et al. (2009) and Henry et al. (2010). Among the objects in these catalogs we reclassified known or proposed SySt: PN ShWi 5 (Miszalski et al. 2009), PN H 2-43 (Belczyński et al. 2000), PN G356.9-05.8 (Zhang \& Liu 2003), and PN G005.2+04.2 (Miszalski et al. 2009). Fluxes of emission lines in SySt were taken from Mikołajewska et al. (1997), GutiérrezMoreno \& Moreno (1998), Pereira et al. (1998) and Luna \& Costa (2005). The D-, S- and D'-type classification of individual stars was adopted from Belczyński et al. (2000) and Phillips (2007). In our study we omitted stars without this classification available. Fluxes of emission lines of both PNe and SySt were reddening corrected using reddening estimates given in the employed catalogs. The Gutierrez-Moreno et al. (1995) diagnostic diagram for PNe and SySt is presented in Fig. 1. Only two SySt would be misclassified as PNe based on this diagram; He 2-172 and $\mathrm{H}$ 1-36. Similar diagrams, using other emission lines are presented in Figs. 2-4.

We investigated known PNe for misclassified SySt in five members of LGGS. In the case of the Small and Large Magellanic Clouds (SMC and LMC, respectively) we employed emission line fluxes reported by Monk et al. (1988) and Leisy \& Dennefeld (2006). In the case of M 81 the Stanghellini et al. (2010) measurements were used. For NGC 300 the fluxes were taken from Stasińska et al. (2013). The M 33 data is from Magrini et al. (2009). The position of extragalactic PNe on the [O III] diagnostic diagram is presented in Fig. 5.

\section{Results}

\subsection{D- and S-type classification}

Most of the SySt are clearly separated from PNe in the [O III] diagram (Fig. 1). Exceptions are two SySt - He 2-172 and H 1-36. Using the Gutierrez-Moreno et al. (1995) criterion for separation of D- and S-type SySt, eight D-type SySt would be misclassified as well as one S-type SySt. Hence, we propose a new criterion for classifying SySt types, namely when [O III] 4363/H $\gamma<1$ and
[O III] 5006/H $\beta<0.8$ the SySt is a S-type system. With this new criterion only two D-type SySt would be misclassified and all of S-type SySt would be classified correctly.

\subsection{Distinguishing between SySt and PNe}

On the Kniazev et al. (2008) diagnostic diagrams, used for separating $\mathrm{PNe}$ and $\mathrm{H}$ II regions, the SySt occupy the same region as PNe (Fig. 2). This is not surprising given the relatively low critical densities $N_{\text {cr }}$ of the [S II] 6717, [S II] 6731 and [N II] 6584 lines. Namely, $\log \left(N_{\mathrm{cr}} / \mathrm{cm}^{-3}\right)=3.2$ for [S II] 6731 line, $\log \left(N_{\mathrm{cr}}\right)=3.6$ for [S II] 6731 and $\log \left(N_{\mathrm{cr}}\right)=4.9$ for [N II] 6584 line, which is significantly lower than $\log \left(N_{\mathrm{cr}}\right)=$ 5.8 for [O III] 5006 and $\log \left(N_{\mathrm{cr}}\right)=7.5$ for [O III] 4363 lines (Appenzeller \& Oestreicher 1988). The only promising diagram is the diagram including [O III] and [S II] lines in which there is a region occupied only by Galactic SySt. However, this region seems to be occupied by extragalactic PNe and H II regions (see Fig. 3 of Kniazev et al. 2008). The difference in line ratios is probably caused by lower metallicity of objects in Kniazev et al. (2008) sample, which caused lower [O III] and [S II] line fluxes compared to H I lines.

We checked various other line ratios and most of the studied diagrams were not helpful in distinguishing between PNe and SySt. The most promising diagrams are presented in Fig. 3. Most of the diagrams included both [O III] lines, which could be helpful when $\mathrm{H} \beta$ and $\mathrm{H} \gamma$ cannot be measured for the GutierrezMoreno et al. (1995) diagram (Fig. 1). Most notably, diagrams with the [N II] 5755 line are the only diagrams without [O III] lines in which there are regions where only SySt are present (Fig. 3). Separation of SySt from PNe could be expected given that the [N II] 5755 line critical density is similar as for [O III] lines, namely $\log \left(N_{\text {cr }}\right)=7.5$ for [N II] 5755 line (Appenzeller \& Oestreicher 1988).

The diagnostic diagrams discussed above are all based on forbidden lines, which can help to distinguish between SySt and $\mathrm{PNe}$ due to the fact that they are good electron density indicators. Another kind of diagnostic diagram, not based on forbidden lines, is the one employing He I lines (Fig. 4). The He I emission line ratios in SySt deviate from Case B significantly due to metastability of the $2^{3} \mathrm{~S}$ level, and they can serve as a diagnostic of density. This is why the He I emission line ratios were used in 

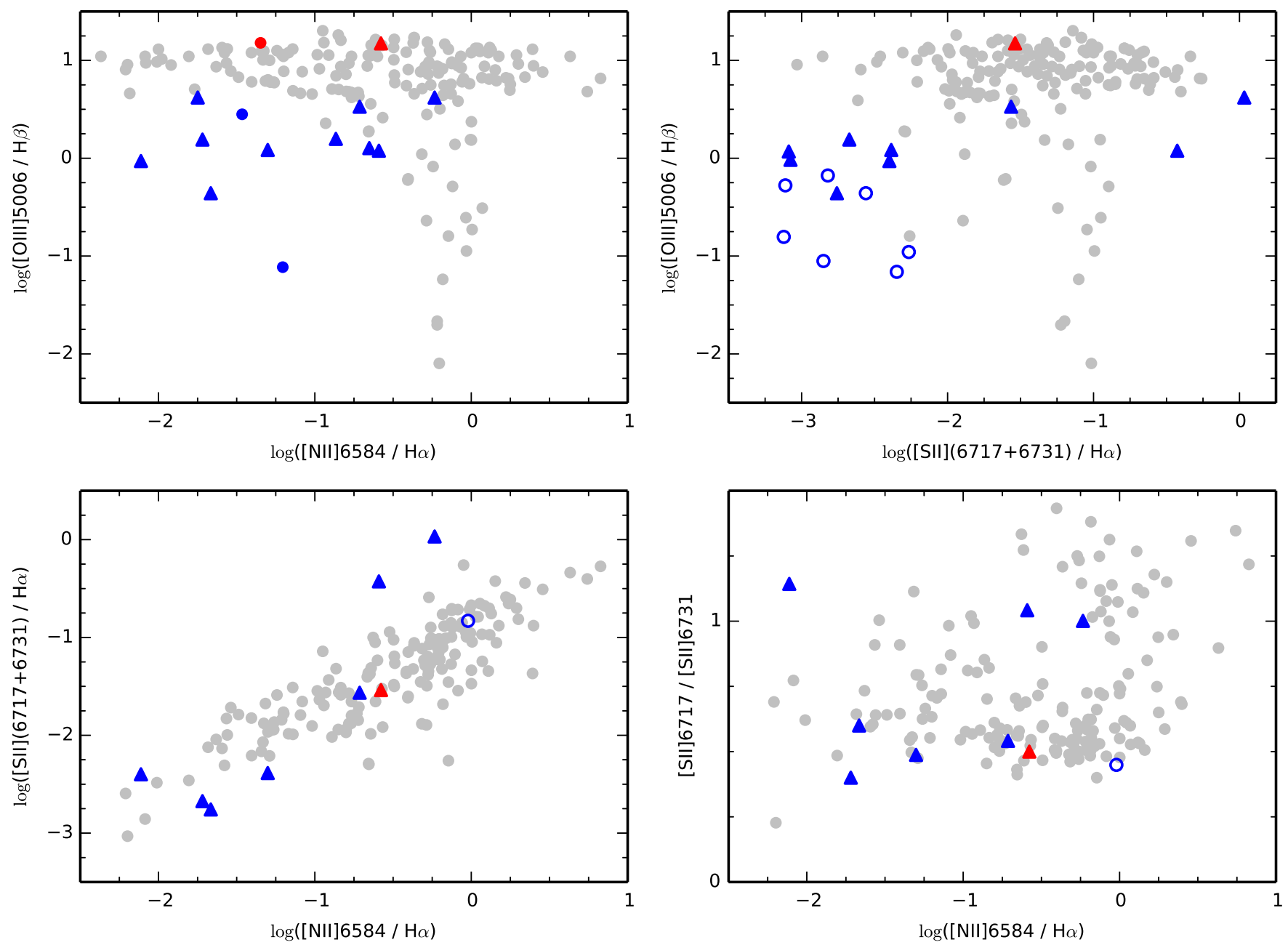

Fig. 2. Kniazev et al. (2008) diagrams used for distinguishing between PNe and H II regions. The SySt are marked with blue symbols and PNe with gray symbols. The SySt that fall into the PNe region in the [O III] diagnostic diagram are marked with red symbols. The S-type SySt are marked with open circles, the D'-type SySt with full circles, and D-type SySt with triangles.

the past to distinguish between D- and S-type SySt (Proga et al. 1994). In our sample of $178 \mathrm{PNe}$, only for $31 \mathrm{PNe}$ the He I fluxes were available. The PNe occupy only a small region in the He I diagram (Fig. 4), while SySt can be found in a much bigger region. The only outlier from the PNe region (Fig. 4) out of the 31 PNe with measured He I lines is PN K 3-90. Out of 123 SySt only 45 can be found in the region occupied by PNe. This shows that the He I diagram can be used as a diagnostic diagram. Moreover, in some SySt the He I emission line ratios are correlated with the orbital phase (e.g., Iłkiewicz et al. 2015), so some SySt can possibly be found in the region occupied by PNe only at certain orbital phase. Furthermore, in some SySt He I lines can be observed only during active phases (e.g., Iłkiewicz et al. 2016).

The He I diagnostic diagram is the most useful new diagnostic diagram. While the separation between PNe and SySt is not as good as in the case of Gutierrez-Moreno et al. (1995) diagram, one of the SySt that would be misclassified as a PNe based on [O III] diagnostic diagram (Fig. 1), He 2-172, would be correctly classified as SySt based on the He I diagram. This is the only diagnostic diagram that would result in correct classification of this system (Fig. 3). The other diagrams could be helpful when the reliable [O III] lines fluxes are not available (e.g., due to saturation), however they are of limited use, since the [O III] lines are more common than the [N II] lines. All of the proposed criteria for SySt are presented in Table 1.

\subsection{SySt candidates among extragalactic $P N e$}

Using the [O III] diagnostic diagram we found six SySt candidates among the LMC PNe (Fig. 5). This is a high number given that only eight SySt are known in the LMC (Belczyński et al. 2000). Out of these six candidates in two of them no He II 4686 line has been detected, in one the He II 4686 emission is relatively weak, and in three the He II 4686 emission is strong (Table 2). The infrared colors (Fig. 6) place only two of these stars near the region occupied by SySt (SMP LMC 93, SMP LMC 104). We conclude that these two are the best candidates for SySt. The other candidates, for which the infrared colors are available, may be unusual PNe, or D'-type SySt with infrared colors more similar to those of PNe.

The effective temperatures of giants in SySt candidates were calculated using the formula, $T_{\text {eff }}=7070 /[(J-K)+0.88]$, from Bessell et al. (1983). The bolometric magnitudes were calculated using the $K$ magnitudes and bolometric corrections, $B C_{K}=-6.75 \log \left(T_{\text {eff }} / 9500\right)$, from Buzzoni et al. (2010). Using the LMC distance modulus of 18.493 mag (Pietrzyński et al. 2013) we were able to calculate the absolute bolometric magnitudes $M_{\text {bol }}$. The results are presented in Table 3 . The calculated effective temperatures are typical for SySt. On the other hand, the absolute bolometric magnitudes for most of the candidates are lower than typical SySt magnitudes (see Fig. 3 

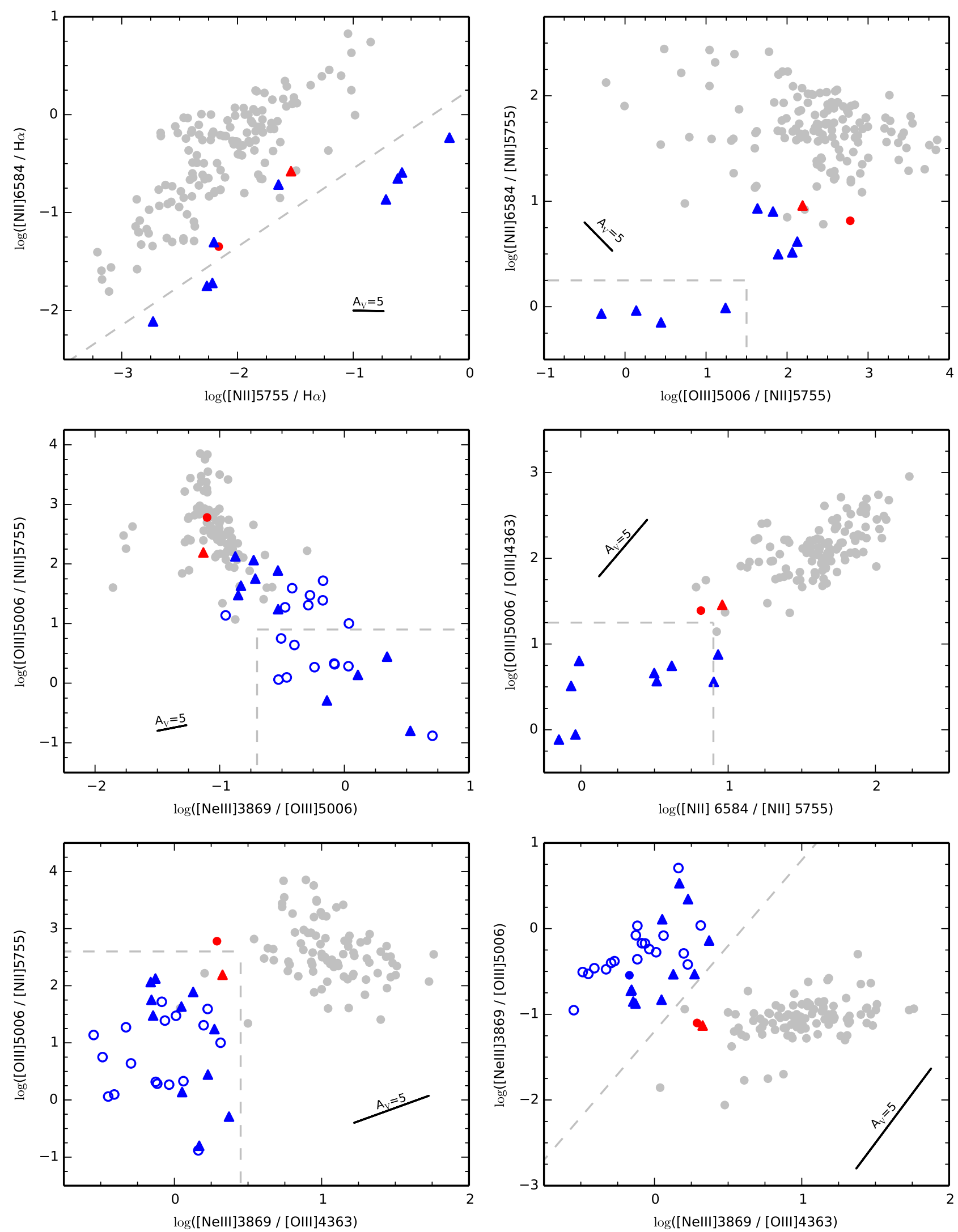

Fig. 3. Various emission line ratio relationships for Galactic SySt and PNe employing forbidden line fluxes. Symbols are the same as in Fig. 2. The reddening was calculated using Cardelli et al. (1989) extinction model with $R_{V}=3.1$ assumed. The gray lines indicate new criteria for SySt proposed in this work.

of Mikołajewska 2007). However, their position on the nearinfrared color-magnitude diagram is consistent with a presence of a low-luminosity RG (Fig. 7). The low luminosity of RGs in these systems could explain why they were not classified as 
Table 1. Proposed criteria for SySt based on emission line ratios.

\begin{tabular}{c}
$\log ([\mathrm{N}$ II $] 6584 / \mathrm{H} \alpha)<0.8 \times \log ([\mathrm{N}$ II $] 5755 / \mathrm{H} \alpha)+0.25$ \\
$\log ([\mathrm{N}$ II $] 6584 /[\mathrm{N}$ II $] 5755)<0.25$ and $\log ([\mathrm{O}$ III $] 5006 /[\mathrm{N}$ II $] 5755)<1.5$ \\
$\log ([\mathrm{O}$ III $] 5006 /[\mathrm{N}$ II $] 5755)<0.9$ and $\log ([\mathrm{Ne}$ III $] 3869 /[\mathrm{O}$ III $] 5006)>-0.7$ \\
$\log ([\mathrm{O}$ III $] 5006 /[\mathrm{O}$ III $] 4363)<1.25$ and $\log ([\mathrm{N} \mathrm{II}] 6584 /[\mathrm{N} \mathrm{II}] 5755)<0.9$ \\
$\log ([\mathrm{O}$ III $] 5006 /[\mathrm{N}$ II $] 5755)<2.6$ and $\log ([\mathrm{Ne}$ III $] 3869 /[\mathrm{O}$ III $] 4363)<0.45$ \\
$\log ([\mathrm{Ne}$ III $] 3869 /[\mathrm{O}$ III $] 5006)>2.0 \times \log ([\mathrm{Ne}$ III $] 3869 /[\mathrm{O}$ III $] 4363)-1.2$ \\
$\log (\mathrm{He}$ I $7065 / \mathrm{He}$ I 5876) $<-0.25$ and $\log (\mathrm{He}$ I $7065 / \mathrm{He}$ I 5876) $<-0.48$ \\
\hline
\end{tabular}

Table 2. Candidate SySt among the extragalactic PNe together with emission line ratios and D- and S-type classification.

\begin{tabular}{ccccccc}
\hline \hline Galaxy & Name & He II 4686/H $\beta$ & {$[\mathrm{O}$ III] 4363/H $\gamma$} & [O III] 5006/H $\beta$ & Ref. & Type \\
\hline LMC & SMP LMC 16 & 0.30 & 1.23 & 7.40 & 1 & $\mathrm{D}$ \\
LMC & SMP LMC 31 & & 0.21 & 0.48 & 2 & $\mathrm{~S}$ \\
LMC & SMP LMC 63 & & 0.10 & 0.24 & 1 & $\mathrm{~S}$ \\
LMC & SMP LMC 93 & 0.62 & 0.23 & 0.48 & 1 & $\mathrm{~S}$ \\
LMC & SMP LMC 104 & 0.62 & 0.44 & 2.00 & 1 & $\mathrm{D}$ \\
LMC & MGPN LMC 31 & 0.64 & 0.53 & 2.59 & 1 & $\mathrm{D}$ \\
M81 & HII403 & & 0.28 & 1.37 & 3 & $\mathrm{D}$ \\
\hline
\end{tabular}

References. (1) Leisy \& Dennefeld (2006); (2) Monk et al. (1988); (3) Stanghellini et al. (2010).

Table 3. Infrared magnitudes of candidate SySt with inferred properties of cool giants.

\begin{tabular}{ccccccc}
\hline \hline Name & $J[\mathrm{mag}]$ & $H[\mathrm{mag}]$ & $K[\mathrm{mag}]$ & Ref. & $T_{\text {eff }}[\mathrm{K}]$ & $M_{\text {bol }}[\mathrm{mag}]$ \\
\hline SMP LMC 16 & 18.09 & 18.03 & 16.94 & 1 & 3500 & 1.39 \\
SMP LMC 31 & 15.777 & 15.362 & 14.111 & 2 & 2800 & -0.78 \\
SMP LMC 63 & 15.789 & 15.907 & 15.052 & 1 & 4400 & -1.17 \\
SMP LMC 93 & 17.077 & 16.497 & 15.767 & 1 & 3200 & 0.44 \\
SMP LMC 104 & 16.994 & 16.076 & 15.128 & 2 & 2600 & 0.46 \\
\hline
\end{tabular}

References. (1) Cutri et al. (2012); (2) Skrutskie et al. (2006).

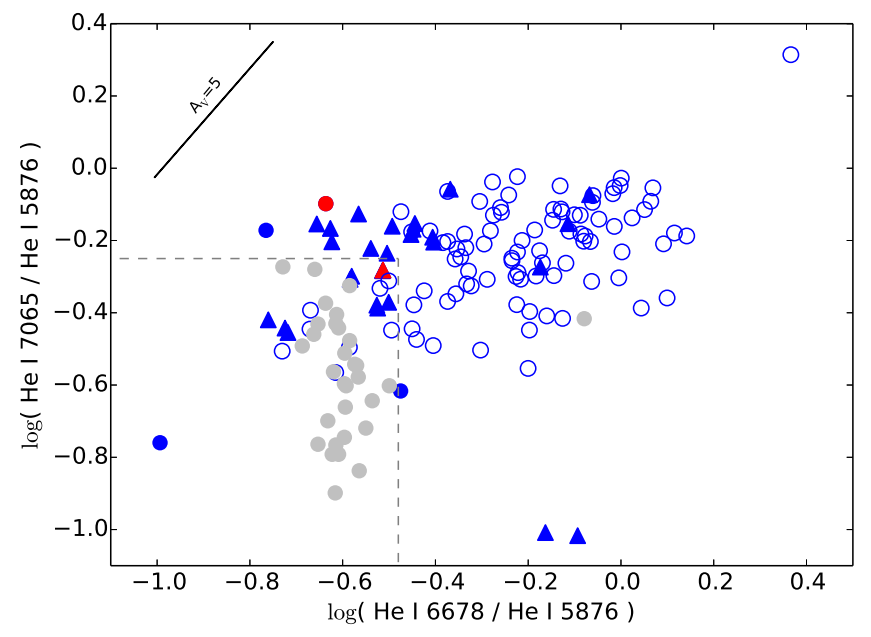

Fig. 4. Same as Fig. 3, but for He I line ratios.

SySt in the past. Moreover, we point out that we did not include reddening correction, the bolometric correction was sensitive to possible errors in deriving the effective temperature, and there could be error in identifying infrared counterparts of candidates.

The only candidate SySt found in other galaxies is HII403 in $M$ 81. If confirmed, this would make this object the first SySt discovered in this galaxy and the farthest known SySt (Gonçalves et al. 2008). However, more observations are needed since it was originally classified as a H II region (Stanghellini et al. 2010).

We stress that deep spectra are needed to confirm the nature of all of the candidates for new SySt. However, objects in the LMC with known $J H K$ magnitudes are most likely SySt or PNe as opposed to other SySt mimics that could reproduce similar spectra and NIR colors. In particular, the most numerous SySt mimics, such as young stellar objects and T Tauri stars, B[e] and classical Be stars as well as Wolf-Rayet stars, (see e.g., Corradi et al. 2008, 2010; Rodríguez-Flores et al. 2014; Miszalski \& Mikołajewska 2014, and references therein) would not have similar position in the color-magnitude diagram as the SySt candidates (Fig. 7).

\section{Conclusions}

In this work we have attempted to find new diagnostic diagrams for distinguishing SySt and PNe. We have also searched for candidate SySt among the known sample of extragalactic PNe. The main results of the work are as follows:

- The only diagnostic diagrams that employ forbidden lines and can distinguish between SySt and PNe are the diagrams with [O III] or [N II] emission lines. This is due to the relatively high critical densities of these lines. A diagnostic diagram with [O III] lines was already known in the literature (Gutierrez-Moreno et al. 1995).

- We propose a diagnostic diagram with He I emission lines as a new tool for distinguishing between SySt and PNe. The 


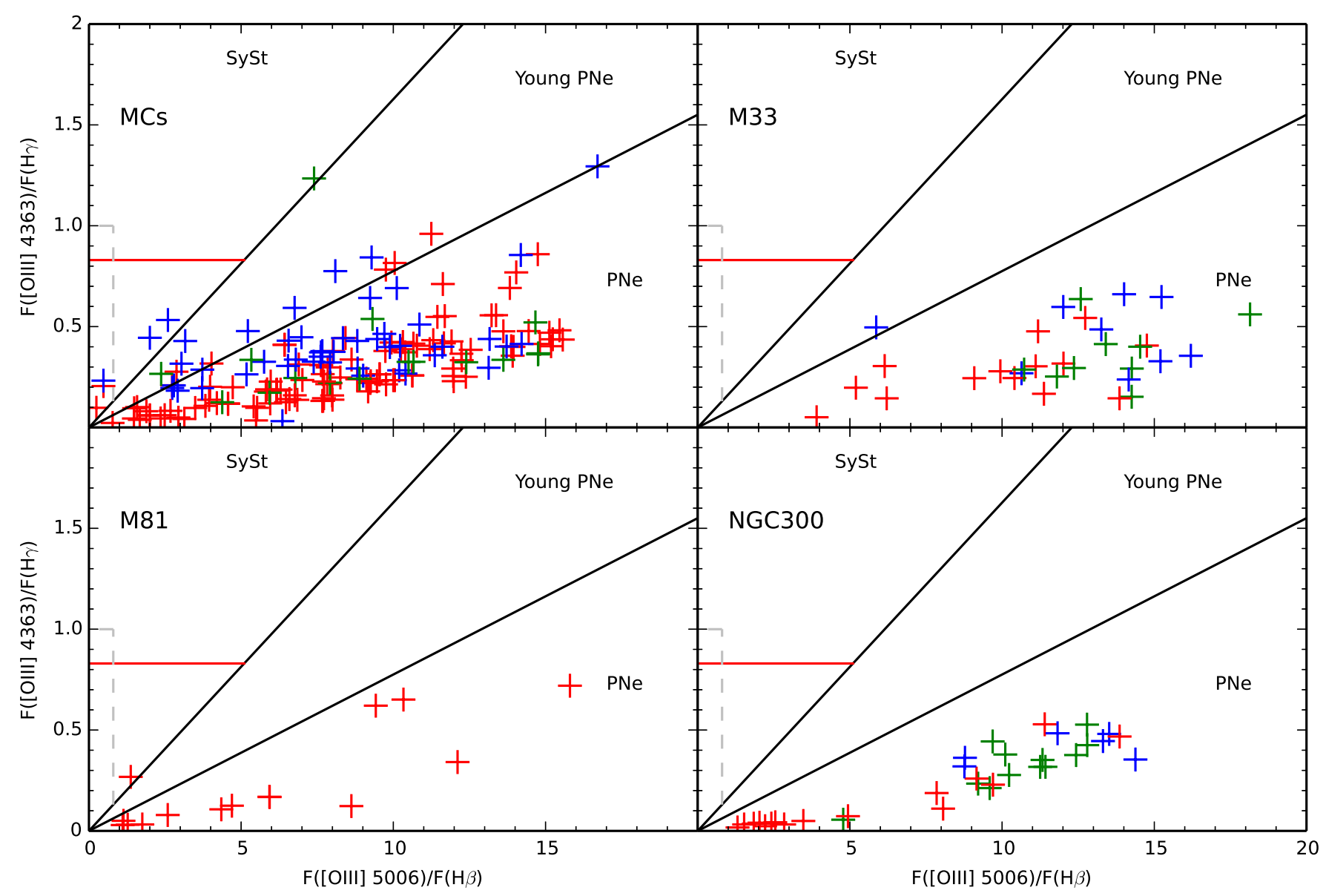

Fig. 5. [O III] diagnostic diagram for candidate SySt among extragalactic PNe with He II 4686/H $\beta$ ratios $>0.3$ (blue points), $<0.3$ (green points), and He II 4686 emission not detected (red points). The red line marks Kniazev et al. (2008) criterion for distinguishing between S- and D-type SySt. The gray lines indicate the regions of S- and D-type SySt proposed in this work.

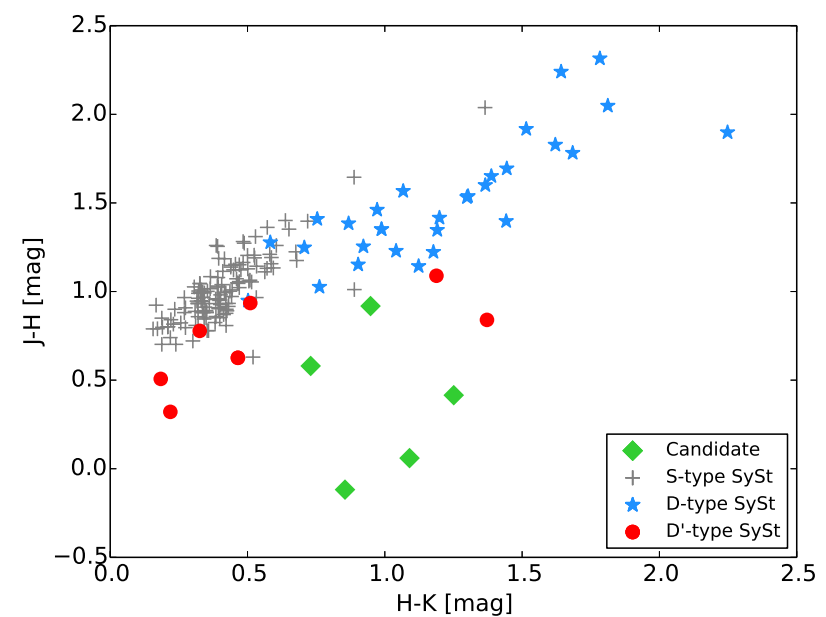

Fig. 6. Infrared colors of the LMC SySt candidates. The SySt candidate colors are from Table 2. The SySt colors are from the 2MASS 6x catalog Cutri et al. (2012).

advantage of this diagram is that it can correctly identify SySt that would be misclassified using the standard [O III] diagnostic diagram. Moreover, the He I diagram has already been used in the past to distinguish between S- and D-type SySt (Proga et al. 1994).

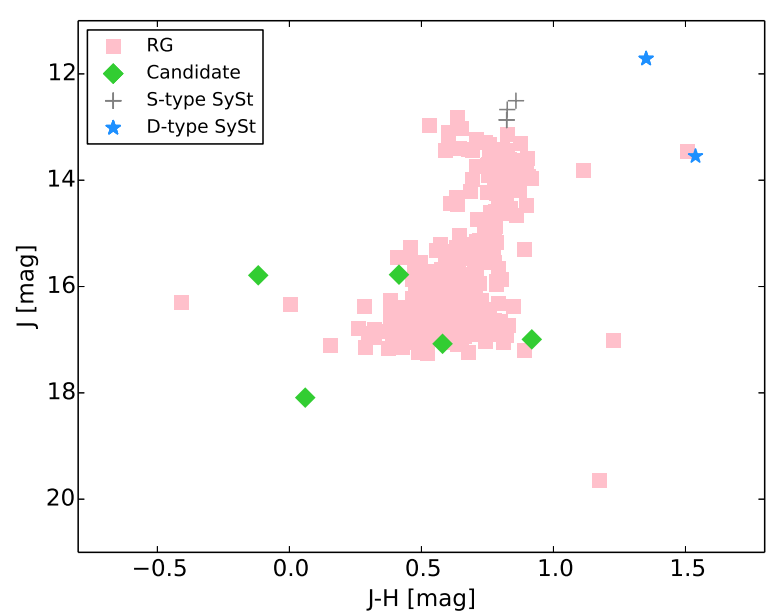

Fig. 7. Position of the LMC SySt candidates and spectroscopically confirmed RGs in the LMC on a near-infrared color-magnitude diagram. The RGs were from (Mucciarelli et al. 2011) and (Carrera et al. 2011). The SySt candidate colors are from Table 2. The SySt and RGs colors are from the 2MASS 6x catalog Cutri et al. (2012).

- All the diagnostic diagrams that are useful for distinguishing between SySt an PNe are electron density indicators, which 
is the physical property that is most useful for distinguishing the ionized nebulae of the two classes of objects.

- We found six candidate SySt in the LMC, while only eight SySt were known in this galaxy thus far. Additionally, we found one candidate in M 81, which if confirmed, would be the first SySt discovered in this galaxy, and the farthest known SySt (Gonçalves et al. 2008).

In future studies it would be useful to search for new diagnostic diagrams in other spectral ranges, where forbidden lines with different critical densities can be found.

Acknowledgements. This study has been financed by the Polish Ministry of Science and Higher Education Diamond Grant Programme via gran 0136/DIA/2014/43. This study has been supported in part by the Polish National Science Centre grant Dec-2013/10/M/ST9/00086. This research has made use of the VizieR catalogue access tool, CDS, Strasbourg, France. The original description of the VizieR service was published in A\&AS 143, 23.

\section{References}

Allen, D. A. 1980, MNRAS, 190, 75

Allen, D. A. 1984, Ap\&SS, 99, 101

Appenzeller, I., \& Oestreicher, R. 1988, AJ, 95, 45

Baella, N. O. 2010, in Stellar Populations - Planning for the Next Decade, eds. G. R. Bruzual, \& S. Charlot, IAU Symp., 262, 307

Baella, N. O., Pereira, C. B., Miranda, L. F., \& Alvarez-Candal, A. 2016, AJ, 151,100

Belczyński, K., Mikołajewska, J., Munari, U., Ivison, R. J., \& Friedjung, M. 2000, A\&AS, 146, 407

Bessell, M. S., Wood, P. R., \& Evans, T. L. 1983, MNRAS, 202, 59

Buzzoni, A., Patelli, L., Bellazzini, M., Pecci, F. F., \& Oliva, E. 2010, MNRAS, 403, 1592

Cardelli, J. A., Clayton, G. C., \& Mathis, J. S. 1989, ApJ, 345, 245

Carrera, R., Gallart, C., Aparicio, A., \& Hardy, E. 2011, AJ, 142, 61

Chen, X., Han, Z., \& Tout, C. A. 2011, ApJ, 735, L31

Corradi, R. L. M., Rodríguez-Flores, E. R., Mampaso, A., et al. 2008, A\&A, 480, 409

Corradi, R. L. M., Valentini, M., Munari, U., et al. 2010, A\&A, 509, A41

Cutri, R. M., Skrutskie, M. F., van Dyk, S., et al. 2012, VizieR Online Data Catalog: II/281

Di Stefano, R. 2010, ApJ, 719, 474

Gonçalves, D. R., Magrini, L., Munari, U., Corradi, R. L. M., \& Costa, R. D. D. 2008, MNRAS, 391, L84

Gonçalves, D. R., Magrini, L., de la Rosa, I. G., \& Akras, S. 2015, MNRAS, 447, 993
Górny, S. K., Stasińska, G., Escudero, A. V., \& Costa, R. D. D. 2004, A\&A, 427, 231

Górny, S. K., Chiappini, C., Stasińska, G., \& Cuisinier, F. 2009, A\&A, 500, 1089 Gutiérrez-Moreno, A., \& Moreno, H. 1998, PASP, 110, 458

Gutierrez-Moreno, A., Moreno, H., \& Cortes, G. 1986, A\&A, 166, 143

Gutierrez-Moreno, A., Moreno, H., \& Cortes, G. 1995, PASP, 107, 462

Hachisu, I., Kato, M., \& Nomoto, K. 1999, ApJ, 522, 487

Henry, R. B. C., Kwitter, K. B., Jaskot, A. E., et al. 2010, ApJ, 724, 748

Iłkiewicz, K., Mikołajewska, J., Miszalski, B., Gromadzki, M., \& Whitelock, P. A. 2015, MNRAS, 451, 3909

Iłkiewicz, K., Mikołajewska, J., Stoyanov, K., Manousakis, A., \& Miszalski, B. 2016, MNRAS, 462, 2695

Kenyon, S. J. 1986, The symbiotic stars (Cambridge and New York: Cambridge University Press), 295

Kniazev, A. Y., Pustilnik, S. A., \& Zucker, D. B. 2008, MNRAS, 384, 1045

Kniazev, A. Y., Väisänen, P., Whitelock, P. A., et al. 2009, MNRAS, 395, 1121

Leisy, P., \& Dennefeld, M. 2006, A\&A, 456, 451

Li, J., Mikołajewska, J., Chen, X.-F., et al. 2015, RA\&A, 15, 1332

Lü, G., Zhu, C., Wang, Z., \& Wang, N. 2009, MNRAS, 396, 1086

Luna, G. J. M., \& Costa, R. D. D. 2005, A\&A, 435, 1087

Magrini, L., Corradi, R. L. M., \& Munari, U. 2003, in Symbiotic Stars Probing Stellar Evolution, eds. R. L. M. Corradi, J. Mikołajewska, \& T. J. Mahoney, ASP Conf. Ser., 303, 539

Magrini, L., Stanghellini, L., \& Villaver, E. 2009, ApJ, 696, 729

Mikołajewska, J. 2007, Balt. Astron., 16, 1

Mikołajewska, J. 2012, Balt. Astron., 21, 5

Mikołajewska, J., Acker, A., \& Stenholm, B. 1997, A\&A, 327, 191

Mikołajewska, J., Caldwell, N., \& Shara, M. M. 2014, MNRAS, 444, 586

Mikołajewska, J., Shara, M. M., Caldwell, N., Iłkiewicz, K., \& Zurek, D. 2017, MNRAS, 465, 1699

Miszalski, B., \& Mikołajewska, J. 2014, MNRAS, 440, 1410

Miszalski, B., Acker, A., Moffat, A. F. J., Parker, Q. A., \& Udalski, A. 2009, A\&A, 496, 813

Miszalski, B., Mikołajewska, J., \& Udalski, A. 2013, MNRAS, 432, 3186

Miszalski, B., Mikołajewska, J., \& Udalski, A. 2014, MNRAS, 444, L11

Monk, D. J., Barlow, M. J., \& Clegg, R. E. S. 1988, MNRAS, 234, 583

Mucciarelli, A., Cristallo, S., Brocato, E., et al. 2011, MNRAS, 413, 837

Pereira, C. B., Landaberry, S. J. C., \& Junqueira, S. 1998, A\&A, 333, 658 Phillips, J. P. 2007, MNRAS, 376, 1120

Pietrzyński, G., Graczyk, D., Gieren, W., et al. 2013, Nature, 495, 76

Proga, D., Mikołajewska, J., \& Kenyon, S. J. 1994, MNRAS, 268, 213

Rodríguez-Flores, E. R., Corradi, R. L. M., Mampaso, A., et al. 2014, A\&A, 567, A49

Schmeja, S., \& Kimeswenger, S. 2001, A\&A, 377, L18

Skrutskie, M. F., Cutri, R. M., Stiening, R., et al. 2006, AJ, 131, 1163

Stanghellini, L., Magrini, L., Villaver, E., \& Galli, D. 2010, A\&A, 521, A3

Stasińska, G., Peña, M., Bresolin, F., \& Tsamis, Y. G. 2013, A\&A, 552, A12

Zhang, Y., \& Liu, X.-W. 2003, A\&A, 404, 545 